\title{
ON THE EXCEPTIONALITY OF SOME SEMIPOLAR SETS OF TIME INHOMOGENEOUS MARKOV PROCESSES
}

\author{
YoICHI OSHIMA
}

(Received July 17, 2000, revised May 10, 2001)

\begin{abstract}
For a Markov process associated with a not necessarily symmetric regular Dirichlet form, if the form satisfies the sector condition, then any semipolar sets are exceptional. On the other hand, in the case of the space-time Markov process associated with a family of time dependent Dirichlet forms, there exist non-exceptional semipolar sets. The main purpose of this paper is to show that any semipolar set $B=J \times \Gamma$ of the direct product type of a subset $J$ of time and a subset $\Gamma$ of space is exceptional if $J$ has positive Lebesgue measure.
\end{abstract}

1. Introduction and preliminaries. Let $X$ be a locally compact separable metric space and $m$ a positive Radon measure on $X$ with full support. In this paper we assume that we are given a family of (not necessarily symmetric) regular Dirichlet forms $\left\{\left(E^{(\tau)}, F\right)\right\}_{\tau \geq 0}$ on $H=L^{2}(X ; m)$ satisfying the following conditions:

(i) For any $\varphi, \psi \in F, E^{(\tau)}(\varphi, \psi)$ is a measurable function of $\tau$.

(ii) For any $T \in[0, \infty)$, there exist positive constants $\lambda_{1}(T)$ and $\lambda_{2}(T)$ such that

$$
\lambda_{1}(T) E^{(\tau)}(\varphi, \varphi) \leq E(\varphi, \varphi) \leq \lambda_{2}(T) E^{(\tau)}(\varphi, \varphi)
$$

for all $\tau \in[0, T]$ and $\varphi \in F$, where $E=E^{(0)}$.

(iii) For any $T \in[0, \infty)$, there exists a positive constant $\Lambda(T)$ such that

$$
\begin{aligned}
E^{(\tau)}(\varphi, \varphi) & \geq 0, \\
\left|E^{(\tau)}(\varphi, \psi)\right| & \leq \Lambda(T) E^{(\tau)}(\varphi, \varphi)^{1 / 2} E^{(\tau)}(\psi, \psi)^{1 / 2}
\end{aligned}
$$

for any $\tau \in[0, T]$ and $\varphi, \psi \in F$ (see [3], [5]).

Although it is not essential to treat our problem, we consider that $E^{(\tau)}$ is defined for any $\tau \in \boldsymbol{R}^{1}$ by putting $E^{(\tau)}=E$ for $\tau<0$. Setting $Z=\boldsymbol{R}^{1} \times X$ and $d \nu(\tau, x)=d \tau d m(x)$, let

$$
\mathcal{H}=L^{2}\left(\boldsymbol{R}^{1} ; H\right)=\left\{u(\tau, x) \mid u(\tau, \cdot) \in H,\|u\|_{\mathcal{H}}<\infty\right\},
$$

where

$$
\|u\|_{\mathcal{H}}^{2}=\int_{\boldsymbol{R}^{1}}\|u(\tau, \cdot)\|_{H}^{2} d \tau=\int_{Z} u(\tau, x)^{2} d \nu(\tau, x) .
$$

Let $E_{\alpha}(\varphi, \psi)=E(\varphi, \psi)+\alpha(\varphi, \psi)_{m}$ and define the norn $\|\cdot\|_{F}$ on $F$ by $\|\varphi\|_{F}=E_{1}(\varphi, \varphi)^{1 / 2}$. Using this norm, define $\mathcal{F}=L^{2}\left(\boldsymbol{R}^{1} ; F\right)$ similarly. For $u, v \in \mathcal{F}$, let

$$
\mathcal{A}(u, v)=\int_{\boldsymbol{R}^{1}} E^{(\tau)}(u(\tau, \cdot), v(\tau, \cdot)) d \tau \text {. }
$$

2000 Mathematical Subject Classification. Primary 60J45; Secondary 60G07, 31C25. 
Identifying $H$ with its dual space $H^{\prime}$, we consider as $F \subset H=H^{\prime} \subset F^{\prime}$. Then $\mathcal{F}^{\prime}=$ $L^{2}\left(\boldsymbol{R}^{1} ; F^{\prime}\right)$ can be defined similarly and $\mathcal{F} \subset \mathcal{H}=\mathcal{H}^{\prime} \subset \mathcal{F}^{\prime}$. For $u \in \mathcal{F}^{\prime}, d u / d \tau$ is considered as a distribution sense derivative of $F^{\prime}$-valued function $u(\tau, \cdot)$. Introduce the space $(\mathcal{W},\|\cdot\| \mathcal{W})$ and a bilinear form $\mathcal{E}$ defined by

$$
\begin{gathered}
\mathcal{W}=\left\{u \in \mathcal{F} \mid \frac{d u}{d \tau} \in \mathcal{F}^{\prime}\right\}, \\
\|u\|_{\mathcal{W}}^{2}=\|u\|_{\mathcal{F}}^{2}+\left\|\frac{d u}{d \tau}\right\|_{\mathcal{F}^{\prime}}^{2}, \\
\mathcal{E}(u, v)= \begin{cases}-\left(\frac{d u}{d \tau}, v\right)+\mathcal{A}(u, v), & u \in \mathcal{W}, v \in \mathcal{F} \\
\left(\frac{d v}{d \tau}, u\right)+\mathcal{A}(u, v), & u \in \mathcal{F}, v \in \mathcal{W},\end{cases}
\end{gathered}
$$

where $(d u / d \tau, v)$ is the canonical coupling of $d u / d \tau \in \mathcal{F}^{\prime}$ and $v \in \mathcal{F}$. For any $f \in \mathcal{H}$, then there exist Markovian resolvents $G_{\alpha} f \in \mathcal{W}$ and $\hat{G}_{\alpha} f \in \mathcal{W}$ such that

$$
\mathcal{E}_{\alpha}\left(G_{\alpha} f, u\right)=\mathcal{E}_{\alpha}\left(u, \hat{G}_{\alpha} f\right)=(f, u) \quad \text { for any } u \in \mathcal{F}
$$

where $\mathcal{E}_{\alpha}=\mathcal{E}+\alpha(\cdot, \cdot)_{\nu}$ (see [6], [10]).

For a family of functions $\mathcal{K}$, we denote the family of non-negative (resp. compact support) functions of $\mathcal{K}$ by $\mathcal{K}^{+}$(resp. $\mathcal{K}_{0}$ ). A function $u \in \mathcal{F}$ is said to be $\alpha$-excessive if

$$
\mathcal{E}_{\alpha}(u, v) \geq 0 \quad \text { for any } v \in C_{0}(Z) \cap \mathcal{W}^{+} .
$$

Denote by $\mathcal{P}_{\alpha}$ the family of $\alpha$-excessive functions in $\mathcal{F}$. Then $u \in \mathcal{F}^{+}$belongs to $\mathcal{P}_{\alpha}$ if and only if $\beta G_{\alpha+\beta} u \leq u v$-a.e. for any $\beta>0$. Given a function $h \in \mathcal{H}^{+}$, put

$$
\mathcal{L}_{h}=\{u \in \mathcal{F} \mid u \geq h, \text { v-a.e. }\} \text {. }
$$

In particular, put $\mathcal{L}_{B}=\mathcal{L}_{I_{B}}$. If $\mathcal{W} \cap \mathcal{L}_{h} \cap \mathcal{P}_{\alpha}$ is non-empty, then there exists a unique function $e_{h}^{(\alpha)} \in \mathcal{P}_{\alpha}$ such that $e_{h}^{(\alpha)} \leq u$ for all $u \in \mathcal{L}_{h} \cap \mathcal{P}_{\alpha}$. Further this function $e_{h}^{(\alpha)}$ is characterized as a minimal function of $\mathcal{L}_{h} \cap \mathcal{P}_{\alpha}$ satisfying

$$
\mathcal{E}_{\alpha}\left(e_{h}^{(\alpha)}, v\right) \geq \mathcal{A}_{\alpha}\left(e_{h}^{(\alpha)}, e_{h}^{(\alpha)}\right)
$$

for any $v \in \mathcal{W} \cap \mathcal{L}_{h}$. In particular, put $e_{h}=e_{h}^{(1)}$ and $e_{B}=e_{I_{B}}^{(1)}$. If $u \in \mathcal{W}$, then there exists a constant $\beta>0$ such that

$$
\mathcal{A}_{1}\left(e_{u}, e_{u}\right) \leq \beta\|u\|_{\mathcal{W}}^{2}
$$

(see [4], [7], [8], [10]).

For a relatively compact open set $A$ of $Z$, define the capacity of $A$ by

$$
\operatorname{Cap}(A)=\mathcal{E}_{1}\left(e_{A}, v\right), \quad v \in \mathcal{W}, \quad v=1 v \text {-a.e. on } A .
$$

It is independent of the choice of $v$ and extended to any compact set $B$ of $Z$ by

$$
\operatorname{Cap}(B)=\inf \{\operatorname{Cap}(A) \mid A \text { is open } \supset B\} .
$$


Further it is also extended to any Borel sets in a usual manner. A function $u$ is called $\mathcal{E}$ quasi-conitinuous if there exists a decreasing sequence of open sets $\left\{A_{n}\right\}$ of $Z$ such that $\lim _{n \rightarrow \infty} \operatorname{Cap}\left(A_{n}\right)=0$ and $\left.u\right|_{Z \backslash A_{n}}$ is continuous. Since $(E, F)$ is a (non-symmetric) Dirichlet form, the $E$-capacity $\mathrm{C}(\Gamma)$ of an open set $\Gamma$ of $X$ can be defined by

$$
\mathrm{C}(\Gamma)=\inf \left\{E_{1}(\varphi, \varphi) \mid \varphi \in F, \varphi \geq I_{\Gamma} m \text {-a.e. }\right\} .
$$

Also the capacity $\mathrm{C}(\cdot)$ is extended to all Borel sets. Further, if we define the capacity $\mathrm{C}^{(\tau)}$ relative to $\left(E^{(\tau)}, F\right)$ similarly, then it follows from (1) that

$$
\left(\lambda_{1}(T) \wedge 1\right) \mathrm{C}^{(\tau)}(K) \leq \mathrm{C}(K) \leq\left(\lambda_{2}(T) \vee 1\right) \mathrm{C}^{(\tau)}(K)
$$

for any $\tau \in[0, T]$. The next result can be found in [6].

THEOREM 1.1. There exists a pair of Hunt processes $\mathbf{M}=\left(Z_{t}, P_{z}\right)$ and $\hat{\mathbf{M}}=\left(\hat{Z}_{t}, \hat{P}_{z}\right)$ on $Z$ such that, for any $f \in \mathcal{H}$, their resolvents $R_{\alpha} f$ and $\hat{R}_{\alpha} f$ are $\mathcal{E}$-q.c.modifications of $G_{\alpha} f$ and $\hat{G}_{\alpha} f$, respectively. Further, if we decompose as $Z_{t}=\left(\tau_{t}, X_{t}\right)$ and $\hat{Z}_{t}=\left(\hat{\tau}_{t}, \hat{X}_{t}\right)$ into the motions $\tau_{t}, \hat{\tau}_{t}$ in $\boldsymbol{R}^{1}$ and $X_{t}, \hat{X}_{t}$ in $X$, then $\tau_{t}$ and $\hat{\tau}_{t}$ are the uniform motion to the right and the left respectively, that is, $\tau_{t}=\tau_{0}+t$ and $\hat{\tau}_{t}=\hat{\tau}_{0}-t$.

It is well-known that, for a Hunt process $Y_{t}$ on $X$ associated with a non-symmetric Dirichlet form ( $E, F)$ satisfying the conditions (2) and (3), any semipolar set is $E$-exceptional, that is, of zero $E$-capacity (see [1], [2], [9]). However, since the space-time process $Z_{t}$ moves to the time direction by uniform motion, the set of the form $\{\tau\} \times \Gamma$ with $m(\Gamma)>0$ is semipolar (in fact, thin) but not $\mathcal{E}$-exceptional, that is, not of zero capacity relative to Cap. The main purpose of this paper is to show the equivalence of the semipolarity and $\mathcal{E}$-exceptionality of the set $B$ of the form $B=J \times \Gamma$ with $|J|>0$, where $|J|$ is the Lebesgue measure of $J$. Different from the process $Y_{t}$, the first hitting distribution $p_{B}$ and the first entry distribution $d_{B}$ of the set $B$ relative to the process $Z_{t}$ is not $\mathcal{E}$-quasi-continuous. But we can see that, for Lebesgue a.e. $\tau \in \boldsymbol{R}^{1}, d_{B}(\tau, \cdot)$ and $p_{B}(\tau, \cdot)$ are $E$-quasi-continuous and $d_{B}(\tau, \cdot)=p_{B}(\tau, \cdot)$ $E$-quasi-everywhere ( $E$-q.e. in abbreviation). The proof of the main result is based upon this property.

The author would like to thank the anonymous referee for careful reading and valuable comments.

2. Equilibrium potentials and hitting probabilities. For a Borel set $B$ of $Z$, define the first entry time $D_{B}$ and the first hitting time $\sigma_{B}$ by $D_{B}=\inf \left\{t \geq 0 \mid Z_{t} \in B\right\}$ and $\sigma_{B}=\left\{t>0 \mid Z_{t} \in B\right\}$, respectively. Using these stopping times, their distridutions $d_{B}$ and $p_{B}$ are defined by

$$
d_{B}(z)=E_{z}\left(e^{-D_{B}}\right) \quad \text { and } \quad p_{B}(z)=E_{z}\left(e^{-\sigma_{B}}\right),
$$

respectively. Clearly, $D_{B}=\sigma_{B}$ and hence $d_{B}=p_{B}$ if $B$ is open. The proof of the next lemma is similar to Stannat [10, Proposition I.3.7] but we shall present it for the readers convenience.

LEMMA 2.1. For any relatively compact open set $B$ of $Z, \lim _{\alpha \rightarrow \infty} \alpha G_{\alpha+1} e_{B}=e_{B}$ in $\mathcal{F}$. 
PROOF. First note that $\lim _{\alpha \rightarrow \infty} G_{\alpha+1} u=u$ in $\mathcal{F}$ for any $u=G_{1} f, f \in \mathcal{H}^{+}$. In fact,

$$
\begin{aligned}
\mathcal{A}_{1}\left(\alpha G_{\alpha+1} u-u, \alpha G_{\alpha+1} u-u\right) & =\mathcal{E}_{1}\left(\alpha G_{\alpha+1} u-u, \alpha G_{\alpha+1} u-u\right) \\
& =\left(\alpha G_{\alpha+1} f-f, \alpha G_{\alpha+1} u-u\right) \rightarrow 0, \quad \alpha \rightarrow \infty .
\end{aligned}
$$

Since $\left\{G_{1} f \mid f \in \mathcal{H}\right\}$ is dense in $\mathcal{F}$, by approximating $e_{B}$ by a function $u$ of the above form relative to $\mathcal{A}_{1}$, it suffices to show that $\mathcal{A}\left(\alpha G_{\alpha} e_{B}, \alpha G_{\alpha} e_{B}\right) \leq k^{2} \mathcal{A}\left(e_{B}, e_{B}\right)$ for some constant $k$. To show this, introduce a kernel $K_{\alpha}$ defined by

$$
K_{\alpha} f(\tau, x)=\int_{0}^{\infty} e^{-\alpha t} f(\tau+t, x) d t=e^{\alpha \tau} \int_{\tau}^{\infty} e^{-\alpha s} f(s, x) d s .
$$

Then it satisfies $(\partial / \partial \tau) K_{\alpha} f(\tau, x)=\alpha K_{\alpha} f(\tau, x)-f(\tau, x)$. In particular, $w_{\alpha} \equiv \alpha G_{\alpha} e_{B}-$ $\alpha K_{\alpha} e_{B} \in \mathcal{W}$.

Since $B$ is relatively compact, there exist $-\infty \leq a<b<\infty$ such that $B \subset[a, b] \times X$. Noting that the function $h(\tau, x)=e^{\tau-a} I_{(-\infty, b]}(\tau)$ satisfies $e^{-t} p_{t} h(\tau, x) \leq h(\tau, x)$, we can see that $e_{B} \wedge h$ is a 1-excessive function dominating $I_{B}$. Hence $e_{B} \wedge h=e_{B}$ and, in particular, $e_{B}(\tau, x)=0$ for $\tau>b$. This further implies that $G_{\alpha} e_{B}(\tau, x)$ and $K_{\alpha} e_{B}(\tau, x)$ vanish for $\tau>b$. Therefore, we have

$$
\begin{aligned}
\mathcal{A}\left(w_{\alpha}, w_{\alpha}\right) & \leq \mathcal{E}_{\alpha}\left(w_{\alpha}, w_{\alpha}\right) \\
& =\alpha \mathcal{E}_{\alpha}\left(G_{\alpha} e_{B}, w_{\alpha}\right)-\mathcal{A}\left(\alpha K_{\alpha} e_{B}, w_{\alpha}\right)-\alpha\left(\alpha K_{\alpha} e_{B}-\frac{\partial}{\partial \tau} K_{\alpha} e_{B}, w_{\alpha}\right) \\
& =-\mathcal{A}\left(\alpha K_{\alpha} e_{B}, w_{\alpha}\right) \leq \Lambda(b) \mathcal{A}\left(\alpha K_{\alpha} e_{B}, \alpha K_{\alpha} e_{B}\right)^{1 / 2} \mathcal{A}\left(w_{\alpha}, w_{\alpha}\right)^{1 / 2} .
\end{aligned}
$$

This implies that $\mathcal{A}\left(w_{\alpha}, w_{\alpha}\right) \leq \Lambda(b)^{2} \mathcal{A}\left(\alpha K_{\alpha} e_{B}, \alpha K_{\alpha} e_{B}\right)$.

Since $E^{(\tau)}(\varphi, \varphi) \leq\left(\lambda_{2}(b) / \lambda_{1}(b)\right) E^{(s)}(\varphi, \varphi)$ for all $\varphi \in F$ and $\tau, s \leq b$, by putting $e_{B}(t)=e_{B}(t, \cdot)$, we can also see that

$$
\begin{aligned}
& \mathcal{A}\left(\alpha K_{\alpha} e_{B}, \alpha K_{\alpha} e_{B}\right) \\
& \quad=\alpha^{2} \int_{\boldsymbol{R}^{1}} d \tau \int_{0}^{\infty} d t \int_{0}^{\infty} d s e^{-\alpha t-\alpha s} E^{(\tau)}\left(e_{B}(t+\tau), e_{B}(s+\tau)\right) \\
& \quad \leq \Lambda(b) \alpha^{2} \int_{-\infty}^{b} d \tau \int_{\tau}^{b} d t \int_{\tau}^{b} d s e^{\alpha(2 \tau-t-s)} E^{(\tau)}\left(e_{B}(t), e_{B}(t)\right)^{1 / 2} E^{(\tau)}\left(e_{B}(s), e_{B}(s)\right)^{1 / 2} \\
& \quad \leq \Lambda(b)\left(\lambda_{2}(b) / \lambda_{1}(b)\right) \alpha \int_{-\infty}^{b} d \tau \int_{\tau}^{b} e^{\alpha(\tau-s)} E^{(s)}\left(e_{B}(s), e_{B}(s)\right) d s \\
& \quad \leq \Lambda(b)\left(\lambda_{2}(b) / \lambda_{1}(b)\right) e^{\alpha b} \mathcal{A}\left(e_{B}, e_{B}\right) .
\end{aligned}
$$

Hence

$\mathcal{A}\left(\alpha G_{\alpha} e_{B}, \alpha G_{\alpha} e_{B}\right)^{1 / 2} \leq \mathcal{A}\left(\alpha K_{\alpha} e_{B}, \alpha K_{\alpha} e_{B}\right)^{1 / 2}+\mathcal{A}\left(w_{\alpha}, w_{\alpha}\right)^{1 / 2} \leq k \mathcal{A}\left(e_{B}, e_{B}\right)^{1 / 2}$ with $k=(1+\Lambda(b)) \sqrt{\Lambda(b)}\left(\lambda_{2}(b) / \lambda_{1}(b)\right)^{1 / 2} e^{\alpha b / 2}$.

LEMMA 2.2. For any relatively compact open set $B, d_{B}=e_{B} v-$ a.e.

PRoOF. Let $h_{B}$ be a measurable version of $e_{B}$ such that $h_{B}=1$ on $B$. Then $p_{t} h_{B}$ is uniquely determined $v$-a.e. independently of the choice of the version $h_{B}$. In fact, if $g=0$ 
$v$-a.e., then from $\left\langle v, p_{t}|g|\right\rangle=\left\langle\hat{p}_{t} 1,|g|\right\rangle_{v}=0$, it follows that $p_{t} g=0 v$-a.e. Since $h_{B} \geq$ $e^{-t} p_{t} h_{B} v$-a.e., there exists a $v$-negligible set $N$ such that

$$
h_{B}(z) \geq e^{-t} p_{t} h_{B}(z) \quad \text { for any } z \notin N \text { and any } t \in \boldsymbol{Q}_{+},
$$

where $\boldsymbol{Q}_{+}$is the family of positive rational numbers. Hence, $\left\{e^{-t} h_{B}\left(Z_{t}\right)\right\}_{t \in Q_{+}}$is a positive supermartingale relative to $P_{z}, z \notin N$. For a finite subset $S$ of $\boldsymbol{Q}_{+}$, if we put $D=\inf \{t \in$ $\left.S \mid Z_{t} \in B\right\}$, it then follows that $h_{B}(z) \geq E_{z}\left(e^{-D}\right), z \notin N$. Letting $S \uparrow \boldsymbol{Q}_{+}$, it follows that $h_{B}(z) \geq E_{z}\left(e^{-D_{B}}\right), z \notin N$. Therefore $e_{B} \geq d_{B}$ v-a.e.

To show the converse inequality, first note that $-(d w / d \tau, h) \leq \mathcal{A}_{1}(h, w)$ for any 1 excessive function $h \in \mathcal{F}$ and $w \in \mathcal{W}^{+}$. Since $d_{B}$ is 1 -excessive and dominates $I_{B}$, to get that $e_{B} \leq d_{B} v$-a.e., it suffices to show that $d_{B} \in \mathcal{F}$. This further follows from the boundedness of $\mathcal{A}_{1}\left(\alpha G_{\alpha+1} d_{B}, \alpha G_{\alpha+1} d_{B}\right)$ relative to $\alpha$. We may assume that $B \subset[0, b] \times X$ for some $b<\infty$. In view of $e_{B} \geq d_{B}$, we have

$$
\begin{aligned}
\mathcal{A}_{1}\left(\alpha G_{\alpha+1} d_{B}, \alpha G_{\alpha+1} d_{B}\right) & =\mathcal{E}_{1}\left(\alpha G_{\alpha+1} d_{B}, \alpha G_{\alpha+1} d_{B}\right) \leq \mathcal{E}_{1}\left(\alpha G_{\alpha+1} d_{B}, e_{B}\right) \\
& =-\alpha\left(\frac{d}{d \tau} G_{\alpha+1} d_{B}, e_{B}\right)+\alpha \mathcal{A}_{1}\left(G_{\alpha+1} d_{B}, e_{B}\right) \\
& \leq \alpha \mathcal{A}_{1}\left(e_{B}, G_{\alpha+1} d_{B}\right)+\alpha \mathcal{A}_{1}\left(G_{\alpha+1} d_{B}, e_{B}\right) \\
& \leq 2 \Lambda(b) \mathcal{A}_{1}\left(\alpha G_{\alpha+1} d_{B}, \alpha G_{\alpha+1} d_{B}\right)^{1 / 2} \mathcal{A}_{1}\left(e_{B}, e_{B}\right)^{1 / 2}
\end{aligned}
$$

Therefore $\mathcal{A}_{1}\left(\alpha G_{\alpha+1} d_{B}, \alpha G_{\alpha+1} d_{B}\right) \leq 4 \Lambda(b)^{2} \mathcal{A}_{1}\left(e_{B}, e_{B}\right)$.

LEMMA 2.3. Let $\left\{B_{n}\right\}$ be a decreasing sequence of relatively compact open sets of $Z$ such that $\operatorname{Cap}\left(B_{n}\right) \downarrow 0$. Then there exists a Lebesgue negligible subset $L_{1}$ of $\boldsymbol{R}^{1}$ and a subsequence $\left\{n_{k}\right\}$ satisfying $\mathrm{C}\left(B_{n_{k}}^{(\tau)}\right) \downarrow 0$ for any $\tau \notin L_{1}$, where $B^{(\tau)}=\{x \in X \mid(\tau, x) \in B\}$.

Proof. By virtue of (5), (7) and Lemma 2.2, since

$$
\int_{\boldsymbol{R}^{1}} E_{1}^{(\tau)}\left(d_{B_{n}}(\tau, \cdot), d_{B_{n}}(\tau, \cdot)\right) d \tau=\mathcal{A}_{1}\left(d_{B_{n}}, d_{B_{n}}\right) \leq \operatorname{Cap}\left(B_{n}\right) \rightarrow 0, \quad n \rightarrow \infty,
$$

there exist a subsequence $\left\{n_{k}\right\}$ and a Lebesgue negligible set $L_{1}$ such that

$$
\lim _{k \rightarrow \infty} E_{1}^{(\tau)}\left(d_{B_{n_{k}}}(\tau, \cdot), d_{B_{n_{k}}}(\tau, \cdot)\right)=0 \text { for any } \tau \notin L_{1} .
$$

In view of (1), we can replace $E_{1}^{(\tau)}$ by $E_{1}$ in (11). Further, since $d_{B_{n_{k}}} \in \mathcal{F}$ and $d_{B_{n_{k}}}=1 \nu$ a.e. on $B_{n_{k}}$, we may assume that $d_{B_{n_{k}}}(\tau, \cdot) \in F$ and $d_{B_{n_{k}}}(\tau, \cdot)=1 m$-a.e. on $B_{n_{k}}^{(\tau)}$ for $\tau \notin L_{1}$. Then (9) implies that $C\left(B_{n_{k}}^{(\tau)}\right) \rightarrow 0$ as $k \rightarrow \infty$ for any $\tau \notin L_{1}$.

LEMMA 2.4. Let $B$ be a relatively compact open set of $Z$. Then there exists a subset $L_{2}$ of $\boldsymbol{R}^{1}$ of zero Lebesgue measure such that $d_{B}(\tau, \cdot)$ is E-quasi-continuous for any $\tau \notin L_{2}$.

Proof. In view of Lemma 2.1 and Lemma 2.2, since $\alpha R_{\alpha+1} d_{B}$ converges to $d_{B}$ relative to $\mathcal{A}_{1}$, there exist a sequence $\alpha_{k} \uparrow \infty$ and a Lebesgue negligible set $L_{3}$ such that

$$
\lim _{k \rightarrow \infty} E_{1}\left(\alpha_{k} R_{\alpha_{k}+1} d_{B}(\tau, \cdot)-d_{B}(\tau, \cdot), \alpha_{k} R_{\alpha_{k}+1} d_{B}(\tau, \cdot)-d_{B}(\tau, \cdot)\right)=0
$$


for all $\tau \notin L_{3}$. Then, by choosing a subsequence $\left\{\beta_{k}\right\}$ of $\left\{\alpha_{k}\right\}, \beta_{k} R_{\beta_{k}+1} d_{B}(\tau, \cdot)$ converges $E$ quasi-uniformly for any $\tau \notin L_{3}$ (see [2, Theorem 2.1.4]). The limit coincides with $d_{B}(\tau, \cdot)$ because $\lim _{\alpha \rightarrow \infty} \alpha R_{\alpha+1} d_{B}=p_{B}=d_{B}$ everywhere. Since $\beta_{k} R_{\beta_{k}+1} d_{B}$ is $\mathcal{E}$-quasi-continuous, for any relatively compact open set $U$ of $Z$, there exists a sequence of relatively compact open subsets $\left\{N_{n}\right\}$ of $U$ such that $\operatorname{Cap}\left(N_{n}\right) \rightarrow 0$ and $\beta_{k} R_{\beta_{k}+1} d_{B}$ is continuous on $U \backslash N_{n}$ for any $k$. By virtue of Lemma 2.3, then there exist a subsequence $n_{\ell}$ and a Lebesgue negligible set $L_{4}$ such that, for $\tau \notin L_{4}, C\left(N_{n_{\ell}}^{(\tau)}\right) \rightarrow 0$ and $\beta_{k} R_{\beta_{k}+1} d_{B}(\tau, \cdot)$ is continuous on $U^{(\tau)} \backslash N_{n_{\ell}}^{(\tau)}$ for any $k$ and $\ell$. Putting $L_{2}=L_{3} \cup L_{4}$, we get that $d_{B}(\tau, \cdot)$ is $E$-quasi-continuous on $U^{(\tau)}$ for any $\tau \notin L_{2}$. Since $U$ is arbitrary, the assertion holds.

3. The main result. Now we are in a position to prove the main result of this paper that any semipolar set $B$ of the form $B=J \times \Gamma$ with $|J|>0$ is $\mathcal{E}$-exceptional. The essential step is the following theorem.

THEOREM 3.1. For any compact set $K$ of $Z$, there exists a Lebesgue negligible set $L$ such that $d_{K}(\tau, \cdot)=p_{K}(\tau, \cdot)$ E-q.e. for any $\tau \notin L$.

PROOF. Let $\left\{B_{n}\right\}$ be a decreasing sequence of relatively compact open sets such that $\bigcap_{n} B_{n}=K$. Then $\lim _{n \rightarrow \infty} d_{B_{n}}(z)=d_{K}(z)$ for all $z \in Z$. Further, since $\mathcal{A}_{1}\left(d_{B_{n}}, d_{B_{n}}\right) \leq$ $\operatorname{Cap}\left(B_{n}\right)$ is bounded, there exists a subsequence of $d_{B_{n}}$ such that its Cesàro means $\left\{u_{k}\right\}$ converges in $\mathcal{F}$ and $d_{K} \in \mathcal{F}$. Then, similarly to the proof of Lemma 2.4 , by choosing a subsequence of $\left\{u_{k}\right\}$ if necessary, there exists a Lebesgue negligible set $L_{5}$ such that $\lim _{k \rightarrow \infty} u_{k}(\tau, \cdot)=d_{K}(\tau, \cdot) E$-quasi-uniformly for any $\tau \notin L_{5}$. In particular, $d_{K}(\tau, \cdot)$ is $E$-quasi-continuous. Since $d_{K} \in \mathcal{F}$, we can see from Lemma 2.1 that $\alpha R_{\alpha+1} d_{K}$ converges to $d_{K}$ in $\mathcal{F}$ as $\alpha \rightarrow \infty$ and hence, there exist a Lebesgue negligible set $L_{6}$ and a subsequence $\alpha_{n} \uparrow \infty$ such that $\lim _{n \rightarrow \infty} \alpha_{n} R_{\alpha_{n}+1} d_{K}(\tau, \cdot)=d_{K}(\tau, \cdot) E$-q.e. for any $\tau \notin L_{6}$. On the other hand, since $\lim _{\alpha \rightarrow \infty} \alpha R_{\alpha+1} d_{K}(z)=p_{K}(z)$, we get the assertion.

LEMMA 3.1. For any compact set $\Gamma$ of $X, \mathrm{C}(\Gamma)=0$ if and only if $\mathrm{Cap}(J \times \Gamma)=0$ for some $J \subset[0, \infty)$ with $|J|>0$.

Proof. Suppose that $\mathrm{C}(\Gamma)=0$. Then there exists a sequence $\varphi_{n} \in F$ such that $\varphi_{n}=1$ $m$-a.e. on a relatively compact neighbourhood $\Gamma_{n}$ of $\Gamma$ and $\lim _{n \rightarrow \infty} E_{1}\left(\varphi_{n}, \varphi_{n}\right)=0$. We may assume that $J$ is a compact set contained in a finite open interval $(a, b)$. Take a non-negative smooth function $\xi$ supported by $[0, T]$ such that $\xi=1$ on $(a, b) \subset[0, T]$ for some $T<\infty$. Then $w_{n}(\tau, x)=\xi(\tau) \varphi_{n}(x) \in \mathcal{W}, w_{n}=1$ v-a.e. on $B_{n}=(a, b) \times \Gamma_{n}$ and

$$
\left\|w_{n}\right\|_{\mathcal{W}}^{2} \leq k_{1}\left\|\varphi_{n}\right\|_{F}^{2} \int_{\boldsymbol{R}^{1}}\left\{\left(\xi^{\prime}(\tau)\right)^{2}+(\xi(\tau))^{2}\right\} d \tau \rightarrow 0, \quad n \rightarrow \infty
$$

for some constant $k_{1}$. Noting that $\left\|e_{B_{n}}\right\|_{\mathcal{F}}^{2} \leq\left(1+\lambda_{2}(T)\right) \mathcal{A}_{1}\left(e_{B_{n}}, e_{B_{n}}\right)$, we have from (5) that 


$$
\begin{aligned}
\operatorname{Cap}\left(B_{n}\right) & =\mathcal{E}_{1}\left(e_{B_{n}}, w_{n}\right) \leq\left\|e_{B_{n}}\right\|_{\mathcal{F}}\left\|w_{n}\right\|_{\mathcal{W}} \\
& \leq \sqrt{1+\lambda_{2}(T)} \mathcal{A}_{1}\left(e_{B_{n}}, e_{B_{n}}\right)^{1 / 2}\left\|w_{n}\right\|_{\mathcal{W}} \leq \sqrt{1+\lambda_{2}(T)} \mathcal{E}_{1}\left(e_{B_{n}}, w_{n}\right)^{1 / 2}\left\|w_{n}\right\|_{\mathcal{W}} \\
& =\sqrt{1+\lambda_{2}(T)} \operatorname{Cap}\left(B_{n}\right)^{1 / 2}\left\|w_{n}\right\|_{\mathcal{W}} .
\end{aligned}
$$

Hence $\operatorname{Cap}\left(B_{n}\right) \leq\left(1+\lambda_{2}(T)\right)\left\|w_{n}\right\|_{\mathcal{W}}^{2}$ and which implies that

$$
\operatorname{Cap}(J \times \Gamma) \leq \operatorname{Cap}\left(B_{n}\right) \leq\left(1+\lambda_{2}(T)\right)\left\|w_{n}\right\|_{\mathcal{W}}^{2} \rightarrow 0, \quad n \rightarrow \infty .
$$

Conversely, suppose that $\operatorname{Cap}(J \times \Gamma)=0$ for some $J$ with $|J|>0$. By virtue of Lemma 2.3 , then there exists a decreasing sequence of relatively compact open sets $\Gamma_{n} \supset \Gamma$ such that $\mathrm{C}\left(\Gamma_{n}\right) \downarrow 0$ which implies that $\mathrm{C}(\Gamma)=0$.

THEOREM 3.2. Let $B=J \times \Gamma$ for some $J$ with $|J|>0$ and $\Gamma \subset X$. If $B$ is semipolar relative to $\mathbf{M}$, then it is $\mathcal{E}$-exceptional.

Proof. It suffices to assume that $B$ is thin, $J$ and $\Gamma$ are compact subsets of $[0, \infty)$ and $X$, respectively. By virtue of Theorem 3.1, there exists a Lebesgue negligible subset $L$ such that $d_{B}(\tau, \cdot)=p_{B}(\tau, \cdot) E$-q.e. for any $\tau \notin L$. Since $B$ is thin, $p_{B}(\tau, x)<1$ for any $(\tau, x) \in B$. On the other hand, since $d_{B}(\tau, x)=1$ for $(\tau, x) \in B$, it follows that $\Gamma$ is of zero $E$-capacity. Hence, from Lemma 3.1, $B$ is of zero $\mathcal{E}$-capacity.

\section{REFERENCES}

[ 1 ] P. J. FitZsimmons, Markov processes and nonsymmetric Dirichlet forms without regularity, J. Funct. Anal. 85 (1989), 287-306.

[2 2 M. Funushima, Y. Oshima And M. TAKeda, Dirichlet forms and symmetric Markov processes, de Gruyter Stud. in Math. 19, Walter de Gruyter \& Co., Berlin, 1994.

[ 3 ] Z. M. MA AND M. RÖCKNER, Introduction to the theory of (non-symmetric) Dirichlet forms, Universitext, Springer-Verlag, Berlin, 1992

[ 4 ] F. Mignot And J. P. Puel, Inequations d'evolution paraboliques avec convexes dépendant du temps, Applications aux inequations quasi-variationelles d'evolution, Arch. Rational Mech. Anal. 64 (1977), 5991.

[ 5 ] Y. Oshima, Lecture on Dirichlet spaces, Universität Erlangen-Nürnberg, 1988.

[ 6 ] Y. Oshima, On a constraction of Markov processes associated with time dependent Dirichlet spaces, Forum Math. 4 (1992), 395-415.

[ 7 ] Y. Oshima, A short introduction to the general theory of Dirichlet forms, Lecture Note in Universität Erlangen-Nürnberg, 1994.

[ 8 ] M. PIERRE, Représentant précis d'un potentiel parabolique, Séminaire Théorie du Potentiel, Université Paris VI, Lecture Notes in Math. 814, Springer-Verlag, Berlin, 1980.

[ 9 ] M. L. SilversteIn, The sector condition implies that semipolar sets are quasi-polar, Z. Wahrscheinlichkeitstheorie und Verw. Gebiete 41 (1977/78), 13-33.

[10] W. Stannat, The theory of generalized Dirichlet forms and its applications in analysis and stochastics, Mem. Amer. Math. Soc. 142 (1999).

DEPARTMENT OF COMPUTER SCIENCE

FACULTY OF ENGINEERING

KUMAMOTO UNIVERSITY

KUMAMOTO, 860-8555

JAPAN

E-mail address: oshima@gpo.kumamoto-u.ac.jp 